\title{
Computational Biology and the Limits of Shared Vision
}

\author{
Annamaria Carusi \\ Oxford e-Research Centre \\ University of Oxford \\ 7 Keble Road \\ Oxford \\ OX1 3QG
}

Annamaria.carusi@oerc.ox.ac.uk

Abstract

Several studies have focused on the social sharing of visual practices as constitutive of evidence within a domain, while there has been relatively less attention paid to points where the social sharing of practices breaks down, or is resisted. This article argues that a study of both types of cases is necessary in order to gain a better perspective on social sharing of practices, and on what other factors this sharing is dependent upon. The article presents the case of currently emerging inter-disciplinary visual practices in the domain of computational biology, where the sharing of visual practices would be beneficial to the collaborations necessary for the research.

Computational biology includes sub-domains where visual practices are coming to be shared across disciplines, and those where instead this is not occurring, and where the practices of others are resisted. A significant point of difference between these sub-domains is between visualisations that render the output of simulations, and those instead which are images taken during observations using the techniques of microscopy. A crossing over, compromise or sharing of practices relating to these different sub-domains is difficult and often resisted. This resistance needs to be contextualised in a far richer account of the relations between the visual artefacts, the scientists who use them within disciplinary domains, the theoretical and instrumentational outlook of the disciplines in questions, and that towards which the science is directed, its domain of study. Social practices alone are not sufficient to account for the shaping of evidence. The philosophy of Merleau-Ponty is introduced as providing an alternative framework for thinking of the complex inter-relations between all of these factors. This philosophy enables us to think of the interconstitutive relations between these different factors, which ultimately define an epistemological and ontological space in which the object of study itself has an active constitutive role, and in which the scientist as person and perceiving body within a knowledge domain is also constituted.

Since the 1980s, several studies of visual perception have persuasively argued that vision is best accounted not by recourse to inner mental representations by rather through socially observable actions and behaviours (e.g. Lynch (1985), Latour (1986), Lynch (1990), Goodwin (1994), Goodwin (1997), Sharrock \& Coulter (1998)). Perception has been dislodged from its position as the basic term in the interface between human beings and their environment and replaced with terms such as 'social practice', and 'vernacular intelligibility'. These theorists claim that we see as we do not because of inner mental representations, nor because of whatever the environment 
may afford us, but because of shared practices whereby groups and communities come to see in the same way. That is, members of groups and communities tend to pick out and perceive the same kinds of entities and processes, and the same kinds of properties and features. In these accounts, the inner world of invisible and mystical mental representations is replaced by the outer world of visible and observable social doings. Often the later philosophy of Wittgenstein is claimed as a precursor of these views, with the notion of language game being interpreted sociologically manner, as a conceptual framework for seeing the meanings of terms, utterances and 'moves' as determined by social agreement alone. Phenomenology generally is another important philosophical precursor, in particular the social phenomenology of Alfred Schutz. The work of Maurice Merleau-Ponty on the phenomenology of perception is less often explicitly drawn upon in this tradition of social accounting for perception but shares many of the same concerns, and is in their intellectual background. One difference with Merleau-Ponty's account is that it dissolves the dichotomy between inner and outer by focusing instead on the perceiving body. This shift in focus allows for an account of perception which sees it as interlinked with the actions of a subject - both actual and possible - relative to an environment which is as 'objective' as it is intersubjective and therefore social. The perceiving body itself is both subject and object of perception, and it is the intertwining and reversibility of these roles which frames the 'answering' of objects in the environment to our shared acts of perception. Rather than shared practices, Merleau-Ponty often speaks of shared modes or 'styles' of perception which are produced through people interacting with each other and with their environments (Merleau-Ponty 1968) ${ }^{1}$.

All of the accounts I have mentioned so far have in common a focus on shared practices, modes or styles in enabling human perceivers not merely to agree on what they perceive, but to perceive the array of objects, events, and other features that are characteristic of human existence. These accounts have also been extended into the more specialised domains of scientific seeing, or, in terms of Charles Goodwin's famous title, of 'professional vision', with several writers showing the ways in which scientific seeing is socially mediated, constructed or constituted through processes which essentially involve shared practices, styles or modes of perception. The central message of these accounts is that knowledge and perception are interdependent and both are dependent on social or communal aspects of science.

Historical, sociological and philosophical accounts of vision and visual practices in professional and scientific domains have yielded fascinating results detailing the variety of ways in which convergence on ways of seeing is obtained through techniques, activities, practices, routines and cultures ${ }^{2}$. More rare are accounts which deal with a break down in sharing, a lack of agreement or downright disagreement, or an inability to come to see as others do, even when the potential for this would appear

An even earlier philosophical account is that of Immanuel Kant who hinted at the central role of shared modes of seeing by invoking the 'sensus communis' or common sense in judgement Although the argument about the sensus communis is to be found in the Third Critique, Kant sees it as an argument relevant to knowledge in general, including the perceptual knowledge which he deals with in the First Critique. See (Carusi: 2008).

${ }^{2}$ The literature on this point is too large to cite. Representative are Lynch \& Woolgar (1990), Baigrie (1996), Jones \& Galison (1998). 
to be quite obvious ${ }^{3}$. Goodwin's 'Professional Vision' (1994) is an interesting example. In this paper, Goodwin contrasts two contexts in which visual practices play a central role in enabling others to come to see. It is not simply coming to see that is at issue, but rather coming to see as someone else sees, in one case, the 'someone else' being the professor, and in the other, the provider of court testimony. The first case is that of archaeology professors training students to 'see' in an archaeological dig; the second is the case where lawyers defending the police officers on trial for the beating of Rodney King use video evidence to convince the jury that the officers' actions did not constitute an abuse. Goodwin sees close analogies between the two cases. He writes: 'It would however be quite wrong to treat the selective vision that is so salient in the King trial as a special, deviant case, merely a set of lawyers' tricks designed to distort what would otherwise be a clear, neutral vision of objective events unambiguously visible on the tape. All vision is perspectival and lodged within endogenous communities of practice.' (1994: 606). The juxtaposition of the King trial with the archaeology professor inducting a student into archaeological field excavation is meant to show the similarities across the two situations, that is the claim that both involve discursive practices which 'shape events' within a field (1994:606), through such practices as coding schemes, highlighting, and picking out domains for scrutiny. Goodwin claims that both of these are cases of instruction despite differences between them, the most important being the fact that the purpose of the two demonstrations puts their audiences in an entirely different position. Whereas in the trial, it is not expected that the audience (the jury) should start annotating, coding, highlighting, etc. for themselves, in the archaeological dig it is expected that the student will carry out the practices for herself, and in fact Goodwin's own analysis focuses on instances when the student is carrying out these practices with the professor (1994: 611-615). The lawyers are not in an 'endogenous community' with the jury; but the students are in that of their professors, or at least are being invited in. Even though the lawyers are presenting what appears to be an ethnography of police practice so that the video evidence can be seen in a different light, this is not a case of instruction of the jury (do they really go away with a better understanding of when beatings are abuses and when they are not, even within so-called 'police practice'?), but rather a rhetorical tour de force on the part of the lawyers, who are able to turn what appeared, at first, to be incontestable video evidence into contestable video evidence, with the result that the first trial did not result in a conviction of the main perpetrator of the violence, whereas the second did.

Goodwin writes that 'The shaping process creates the objects of knowledge that become the insignia of a profession's craft: the theories, artifacts and bodies of expertise that are its special and distinctive domain of competence' (1994:606). While accepting much of Goodwin's account of the ways in which shared vision operates, as well as the overall project of turning away from mentalistic accounts of perception towards more socially embedded accounts, in this paper I turn attention to their limits,

\footnotetext{
${ }^{3}$ Peter Galison's (1997) account of the image and logic traditions of experimental and theoretical physicists is a notable exception, showing how these traditions struggled - and sometimes still struggle - to come to mutual comprehension. His notion of the trading zone as a way for scientists to come to sufficient understanding so that they can at least collaborate in a mutually beneficial way is useful; however, it sees scientists as interacting primarily through language, or gives a linguistic metaphor of their interactions, claiming that scientists in interdisciplinary domains develop pidgins and creoles which allow them to operate in the trading zone. This leaves out of account the role of ways of seeing in the emergence of those trading zone languages, which seems to be a lacuna in an account of these deeply visual cultures.
} 
in particular with respect to two points. The first is that accounts of social shaping of vision need to be able to distinguish between a sharing -via demonstration and other means - of visual practices which aims to enable others to use the practices themselves and potentially disagree; and a 'sharing' - even ostensibly using the same means - which aims instead to disenable others in some way, by undermining or removing the possibility of disagreement. In the first instance, a demonstration of a practice aims to create an equal discursive partner; in the second, this is not the case: rather the aim is closer to silencing the audience, in-capacitating them in some way. The student trained to be an archaeologist will come to share practices of discerning evidence which will one day put her in a position, at least potentially, to contest the views of her professor, and even, possibly, the practices. The jury is not in this position. Even though they may contest the evidence, it is not in virtue of the lawyers sharing their practices with them, but rather by resisting that apparent sharing. If a detailed account of the social situations such as that given by Goodwin does not allow us to tell the difference, this does not mean that there is not a difference.

The second point is related to the first, and concerns those points where attempts to share visual and other practices meets resistance of greater or lesser robustness. What is the source of this resistance and what does it tell us about the limits of socially sharing practices? To pursue this line of enquiry it is necessary to focus on cases where practices are not shared and where there is real resistance to their being shared, as much as where they are shared. The aim of this paper is not to contest the close relationship between shared visual practices and the constitution of evidence, and therefore, the justification of claims made in specific domains; but rather to explore cases where sharing is more problematic, in order to understand better their significance for an overall account of evidence as socially shaped. Emerging interdisciplinary domains of scientific research are generally good places to look for such examples. In this paper, computational biology is presented as just such an example. It is a superb field of enquiry for this exploration, since it offers examples of both types: that is, where visual artefacts and some associated practices do come to be shared, and those, instead, where they are not shared, and are even contested. In order to understand these practices, they must be placed in the broader epistemic context in which they occur. In order to understand the visual practices around observation, and where they are shared as opposed to where they are not, it is important to look at the settings in which they occur: their history, and the locations in which the practices occur (for example, the 'dry lab' of in silico experiments as opposed to the 'wet lab' of laboratory experiments), and the contexts in which the visual artefacts are used, and how. We need to ask how computational biology is re-constituting the field of biology and what counts as a biological question, for what ends or purposes these questions are asked, and what counts as an answer. Whereas computational biology uses mathematical and computational approaches, and is predominantly quantitative in nature, many sub-domains within biology use predominantly qualitative approaches. Despite the differentiation of approaches along the quantitative / qualitative distinction, observation has a central place in both approaches, though what is observed and the practices which surround it are distinct: a simulated biological process on one hand or an actual biological process on the other. This leads to a radically different, and possibly irreconcilable set of visual practices, since they are directed towards fundamentally different entities. A reconciliation of visual practices would imply a change in the nature of the field studied. 
This paper uses the emerging domain of computational biology to explore those points where visual practices are not shared and seemingly cannot come to be shared without a deep change across the entire epistemic framework of the domain. The point of the exploration is to test the limits of accounts of specialised seeing as socially shaped, and a consideration of what other factors play a role in shaping perceptions in a field of specialised vision.

The case described in this paper centres on two groups of Computational Biologists: one which can more precisely be described as computational physiology, and which focuses on the modelling and simulation of processes relating to organs, and one which focuses instead of processes relating to multicellular systems. The modelling and simulation of electrical currents across the heart is a paradigm case of the first, whereas that of the growth of specific types of tumour cells is a paradigm case of the second. The case study emerges out of ongoing participative research with members of both groups and includes observations, interviews, participation at workshops and seminars, my teaching of research skills in the context of a doctoral course for computational biology students. A Forum on Scientific Method in Biology was held as part of this study: this was an event which invited a small group of biologists 'of different persuasions' to discuss with each other what biological method is, with the aim of understanding which methods are considered to be likely candidates for arriving at specifically biological questions and answers. ${ }^{4}$ Accounts of biological practice from the history of science and of modelling and simulation from the philosophy of science have also been drawn upon.

The case study is set out in the following sections: The first section sets the background of computational biology as an emerging interdisciplinary domain in biology; the second section describes the form of collaboration that needs to occur between different disciplines for computational biology to develop as a programme of research; in the third section the visualisations that result from the modelling and simulation process are focused upon as visual artefacts around which convergence and divergence occur, while in the fourth section, the observations and images relating to microscopy are discussed. The convergences and divergences in both sets of practices are elaborated upon in the fifth section. In the final section, conclusions concerning the sociality and epistemology of visual practices in contested domains are drawn.

\section{Section 1: Computational Biology as a programme of research}

Computational Biology can be defined as the application of computational methods to the study of biological processes, specifically the methods and techniques of modelling, simulation and visualisation. Computational Biology is closely related to Systems Biology, Mathematical Biology and increasingly, Synthetic Biology. It is a multi-disciplinary area of study, drawing on the expertise and knowledge of a wide array of disciplines: computer scientists, numerical analysts, engineers, mathematicians, biophysicists and a broad range of the life sciences, physiologists, developmental biologists and others. Computational biology requires cross-

\footnotetext{
${ }^{4}$ The Forum was convened by Annamaria Carusi (philosopher), Blanca Rodriguez (computational biologist) and James Wakefield (cell biologist); other participants were: Brian Goodwin, Denis Noble, Evelyn Fox Keller, Kevin Burrage, Philip Maini, Lynn Margulis, Derek Terrar, Eric Werner, Tim Horder. A full transcript of the Forum can be found here: [url to be supplied]
} 
disciplinary collaborations in order to be successful. At the heart of computational biology are models and simulations; simulations are also referred to as in silico experiments, in contrast with in vivo experiments.

In a bottom-up approach to modelling, data for modelling is acquired from laboratory experiments. The experiments supply the parameter values on the basis of which a mathematical model is built. The model attempts to give a quantitative explanation of the phenomena observed in the experiment: that is, it tries to give an account of the mechanisms which make it the case that the parameter values are as they are. The model is an hypothesis, which is tested at two points: the simulation allows a testing of different solutions, the result of which is a refinement of the initial hypothesis concerning the underlying mechanism. Crucially, this hypothesis must be tested in wetlab, in order for the model to be validated. Models can also be built as theoretical entities without any input from experimental data to start off with. This is a more topdown approach to modelling, which must, however, eventually be validated or refuted by experiment. Without experimentally generated parameter values models have no reality: either in their original formulation nor in their final applicability.

While there is not yet general consensus among the disciplines involved concerning the epistemic status of mathematical models, one view is that mathematical modelling is a hypothesis generating (and therefore predictive) device; a mathematical model, however, is not computational in itself, and can just as easily be worked out using pen and paper. The mathematical model is in the form of algebraic equations; for it to be solved, numerical analysis and computational algorithms are used to establish the possible numerical values of the derivatives. This is carried out computationally in a simulation.

The success of computational biology depends on there being a close connection between in vivo and in silico experiments, or wetlab and drylab. Experiments conducted in wetlab are crucial at two points of the research cycle: in order to supply data for the construction of models, and in order to test the models. The models can be seen as hypotheses regarding specific biological processes (to be discussed further in section?). A successful computational biology research programme depends on there being a constant flow of data from wetlab to drylab and back. This involves a complex set of interactions and collaborations between team members of different disciplines and backgrounds. Modellers and simulators typically have a background in physics, mathematics, or computer science, while experimentalists come from a broad range of biological sciences: physiology, biochemistry, developmental biology, etc. It is typical of even successful collaborations that team members have differing conceptions of the research. For example, there are different understandings of models and modelling, and even of which part of the research process is the strictly modelling part of it, with aspects of simulation sometimes being seen as modelling and sometimes not.

However, collaborations are not always successful, since computational biology is not universally accepted as a positive approach and methodology in biological research. The difference in the acceptance and ease of collaboration can result from differences

\footnotetext{
${ }^{5}$ And in fact, is more likely to be worked out in this very low tech fashion as was discovered in our investigation into the working practices of the Integrative Biology team members (Mascord, Jirotka \& Carusi 2007).
} 
in the 'stages of development' of the research programme in the various pockets of biological research, although this assumes that all biological sciences will eventually be 'mature' enough to develop into the use of full-fledged computational methods. A brief history of heart and cancer modelling goes some way to explaining why collaborations in the former are relatively easier than collaborations in the latter.

Heart modelling has a longer history than cancer modelling, going back to the Hodgkin and Huxley model of electrical stimulation in excitable cells such as neurons and cardiac myocytes, in 1953. Denis Noble modified the model so that it could also be used 'to describe the long-lasting action and pace-maker potentials of the Purkinje fibres of the heart' (Noble 1962: 318). Since then there has been an extensive experimental programme generating data to parameterise the models, and to validate them. For a variety of reasons, primarily having to do with differences between whole-organ and cellular processes and their tractability to understanding through mathematical approaches, and the only relatively recent development of mathematical and computational techniques capable of dealing with the levels of complexity of biological processes, cancer modelling is at a much earlier stage of development.

In the heart modelling community, there is a long-established collaboration between experimental physiologists, mathematicians and computer scientists. Heart modelling and in silico experiments started off with individuals who embodied interdisciplinarity in that they were experimental physiologists, and therefore already geared towards the quantitative analysis of experimental data. There has been a long tradition of experimental physiologists who knew enough mathematics to develop their own models. As they conducted the experiments themselves, they were also able to test the models themselves: there was no gap between the development and testing of a model. As the models became more complex, professional mathematicians began to work in the area, and as there was a need to run far more complex algorithms at a greater speed, professional computer scientists also began to collaborate. However among mathematicians, computer scientists and physiologists there was at least a shared understanding of the need for the collaboration, and a shared commitment to the use of quantitative methods, at least alongside observational methods. This creates a natural collaboration between experimental physiologists and mathematicians / computer scientists. Because of the increasing complexity of the problems and the need to speed up the algorithms, truly interdisciplinary individuals become scarcer, and there is a greater need for distribution of expertise. Cooperation and collaboration are pre-requisites for progress (Welsh et al 2006), and have been in place in some instances at least since the 1980s.

Whereas computational and mathematical approaches to whole organ processes (such as electrical currents in the heart) require collaboration with physiologists, in the domain of tumour growth and other multicellular processes, collaboration with cell biologists is required. However, cancer modelling and cellular biology generally have a much shorter history of using quantitative techniques, with fewer people able to span the divide between biology and mathematics, in view of the greater complexity of the biological processes and thus also of the mathematics. Thus, even when there are fully interdisciplinary practitioners, such as Marc Kirchner, there are fewer of them, and they are harder to follow. Therefore, there are not the interdisciplinary individuals who can spearhead the programme from within the biological domain. The corollary of the absence of an overlapping space has been the entrenchment of 
very different epistemic cultures, one of which is predominantly qualitative, whereas the other is predominantly quantitative. This makes co-operation and collaboration far more difficult to achieve. Indeed, it can even be questioned whether this is computational biology, since the main work is still being carried out at the level of mathematical modelling itself and how it can be used for simulation (which is why there are many more mathematicians rather than computational researchers of different sub-disciplines involved in this area).

The computational biology programme of research is still relatively young, and is still a programme in need of validation and acceptance in mainstream science. While it has been successful in garnering funding, with several large funding programmes internationally devoted to it ${ }^{6}$, it has not met with universal approval, and sometimes struggles to find the collaborators. However, because interdisciplinary collaboration is crucial for all aspects of the computational biology programme, a great deal of energy is invested into it. Thus, the computational and mathematical researchers, from doctoral to professorial level, involved in the cancer modelling programmes are well aware of the need to cultivate potential partners in biology, and spend a great deal of time in PR-type exercises. A number of strategies are used, including inter-personal meetings with potential partners, workshops geared towards biologists, opportunities for joint supervision of students, and so on. Biologists are the implicit audience of several journal articles by mathematical modellers describing what modelling can contribute to cancer research, and use several persuasive means to get biologists on board. For example Byrne et al (2006) put forward an argument for the use of mathematical models in studying tumour growth which appeals to the shared value of finding a cure for cancer: 'In this paper, we review a number of mathematical models that have been developed to describe some of the above aspects of tumour growth. In so doing, we aim to show how mathematical modelling, computation and analysis can generate useful insight into the mechanisms that underpin this devastating disease' (2006: 1564) ${ }^{7}$.

While important in terms of the overall strategy of computational biology, these discursive strategies remain relatively external to the characteristic practices of the computational and mathematical modellers. Visual persuasive appeals are also used and to some extent the use of visualisations can be seen in a similar vein, as essentially rhetorical communications. As will be discussed in the third section, the modelling community will often use a style of visualisation with which experimentalists are familiar in order to establish common ground. However, this visualisations have a more significant role in establishing this programme of research.

\section{Section 2: Computational Biology as an interdisciplinary collaboration}

For the mathematicians and computer scientists in the project, it is essential that the models they develop be parameterised with data derived from wetlab experiments. Crucially, experiments must be carried out to supply the parameter values in the terms required by the models and in silico experiments, and they regularly are, in a mature

\footnotetext{
${ }^{6}$ With projects such as the European funded Network of ExcellenceVirtual Physiological Human.

${ }^{7}$ Other articles along the same lines for mathematical modeling in cancer is Komarova (2005), Van Leeuwen et al (2007); one with greater emphasis on experimental physiology is Kohl et al (2000); Hunter \& King (2003); for the most recent development of the computational biology programme towards simulating all organs of the human body: Fenner, Brook et al (2008)
} 
programme of research such as heart modeling. Data provided by experiments for the modelling process are labelled with the term 'parameter values'; however the term can mean different things for a mathematical computational biologist than it does for a biologist or physiologist. Data in the form of parameter values is the only way for models to 'gain reality', by which is meant for them to engage with actual physical processes, and to have a chance of being tested, and possibly validated. A 'healthy' research programme in this domain is indicated by a constant flow of parameter values, from in vivo experiment, to model, to in silico experiment, and back. It is essential to keep this flow going, as continuously and smoothly as possible. A parameter value is a quantitative entity - it can relate to temporal or spatial properties, or concentrations, speeds, and so on.

This is not so for other areas of biology of interest to mathematical biology, such as cancer modelling. Several mathematicians in the study expressed frustration with biologists for not carrying out the required experiments, and commented on the difficulty of extracting parameter values from biologists. The difficulties relate to agreeing which parameters may be of interest, as well as the meaning of the values: for example, whether they are relative or absolute values. For a biologist, it may be sufficient to know that there is a relation between two (or more) properties, and they are interested in the quantity of these only relative to each other. Mathematical biologists, instead, must know 'absolute' quantities, concentrations, etc. of these properties: that is, what they are independently of each other. Only with this knowledge, can they begin to pinpoint what they see as the causal mechanisms of the processes they are attempting to understand.

As a computational mathematician put it:

... biologists often just want to know that something happens, two proteins interact which regulate the expression of some other protein [...] now, that's it, they just want to know that that's the mechanism. We want to know exactly how much of that protein has to be present in order to cause exactly what degree of regulation of this other protein ... so we can build a model which explains the concentration of the thing that caused the regulation of that other thing [...] [by] a series of graphs [and a] model to reproduce those graph. Biologists don't and can't typically do that. They just know that these proteins are present in the system. They just tell you they're there. No concentrations, rate constants, absolute concentrations in any particular situation. Biologists either can't do that or they won't because it's not what they're interested in.

A very important aspect of this relationship is the fact that the research questions of computational biologists are often geared towards interventional strategies - for example, medical or pharmaceutical interventions. Computational biology for the understanding of cardiac disease is closely associated with pharmaceutical research. For example, the mathematician quoted above went on to say:

I want to be able to quantitatively explain what's going on in terms of molecules, forces, exactly what's going on. And the reason I want to explain that is I want to design new treatments and drugs and only way to do that safely is to be able to say exactly what's going on. 
But this involves testing for parameters that may be of little interest to biologists who are not medical, clinical or pharmaceutical researchers. This is also found in contexts where collaborations with biologists are sought for engineering purposes, generating many questions about molecular kinematics for the engineers in the collaboration, but for which 'it remains far from obvious in what sense they are biological questions' (Keller 2002: 232). This is a crucial point of difference in the epistemic goals of these disciplines.

\section{Section 3: Visualisations in the context of modelling and simulation}

Computational biology in the domain of whole organ modelling is a domain where convergence has occurred to a sufficient point to allow for a collaborative interdisciplinary programme of research. The collaboration between experimental physiologists and computational biologists is greatly facilitated by the fact that they share a common vocabulary of parameter values: that is, not only what they mean, but what values it is useful and desirable to test in an experiment. This convergence around parameter values is made possible by the fact that they hold research questions in common, and thus their research is driven by the same interests and purposes --including for example, seeking collaborations with industry partners such as pharmaceutical companies. The flow from wetlab to drylab and back is kept going through common research projects, dedicated doctoral programmes, co-supervision of doctoral students and the increasing numbers of interdisciplinary researchers. The willingness of experimentalists to supply data for the simulations is a sign of the usefulness of the results of the simulations for their own research questions; and indeed, both groups converge on collaborations with external industry partners, in particular pharmaceutical companies which are increasingly interested in using computational biology techniques for drug discovery and testing ${ }^{8}$. Convergence on this external industry collaboration also indicates agreement on the purpose (or at least $a$ purpose) of research; and this implies also agreement on a shared meaning of 'parameter value'" There is coordination, but not complete overlap between the different disciplines, where there are still distinct roles for the different disciplines, as well as much disagreement regarding, for example, what a model is, or even what computational biology as a field is, and what exactly it is trying to do. While there is some discussion, there is not disagreement to the extent that it prevents the collaboration from occurring at all.

The key role of visualisations in the computational biology programme of research is evident in several ways: the visualisations are prominent in teaching, in workshops and in publications. With the aim of getting experimentalists on board, the visualisations are a powerful communicative tool in conveying complex mathematical ideas in a visually compelling way. In virtue of this strong communicative aspect they also play a rhetorical and persuasive role in this domain, with frequent uses as a means of 'selling' the programme to potential collaborators and funders. The visualisations are also cultural identifiers for computational biology groups, being used as logos, office artworks and the like.

\footnotetext{
${ }^{8}$ For example in the PreDICT project [url to be supplied].

${ }^{9}$ In Galison's terms, these would be candidates for pidgin/creole terms.
} 
However, this must not distract attention from the epistemic roles of visualisations. They are used for data analysis, as tools of discovery and exploration of the processes under study; they are used for evidence and justification and claims. Visualisations are not an optional add-on to modelling and simulation; they are not an illustration of concepts and statements that are also couched in verbal terms. Rather, they are an integral part of the simulation process. As Winsburg puts it:

Visualization is by far the most effective means of identifying characteristic features out of complex dynamical data sets, and so it is the most, if not the only, effective means of judging the degree of calibration a simulation enjoys with other data sets and with analytic results. Thus, visualization plays a crucial role in sanctioning as well as in analyzing simulation results. Not only does the epistemology of simulation call upon resources that are empirical, and that come from outside of the theory, it also calls upon the faculties of the observer (1999:290).

A visualisation of a simulation is a perceptual concretisation of the abstract mathematical model that is being simulated; in it, scientists are able to observe the mathematically expressed causal mechanisms that they have posited and tested in the simulation.

The research strand devoted to visualisations is a central one in the overall computational biology programme, with doctoral dissertations and other research being devoted to it. The process of getting from MRI images, or from histology to whole organ visualisations is a research challenge in its own right (Burton et al 2006; Plotkowiak et al 2008), and is demonstrated in Figure 1.

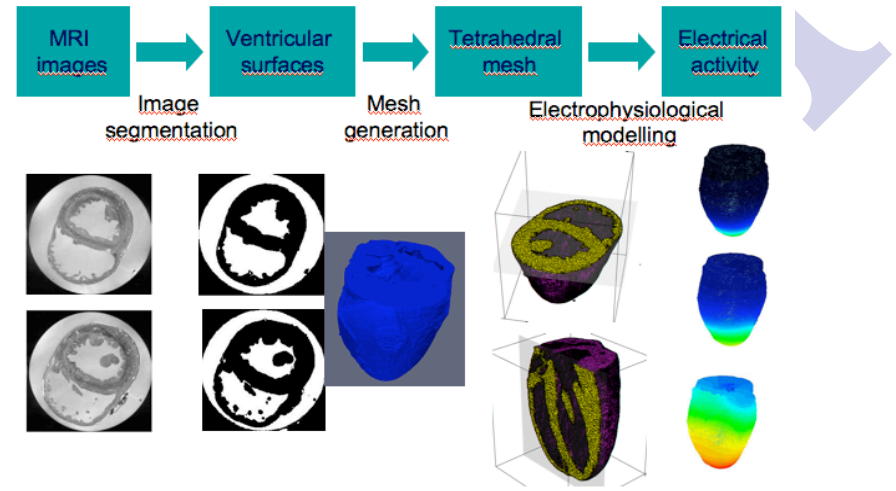

Figure 1 From images to visualisations via Tetrahedral Meshes

For the purposes of my argument, it is important to notice two things about the visualisations: first, they bring about a common mode of perception and observation which is the fulcrum around which this newly forged epistemic culture of computational biology is forged, and second they are a qualitative rendering of a quantitative scientific process.

On the first point: In a previous paper (Carusi: 2008), I have argued that the software that comes to be used for the visualisations within a research group or a research collaboration bring about a common style and mode of perception among disparate 
groups. A good example of this is the collaboration between physiologists and computationalists. Physiologists make use of various imaging techniques, including microscopial images, MRIs, fMRIs, and make extensive use of images in their research. An image, however, is a very different representation than a visualisation, since it has an entirely different causal history. Because of this, the two visual artefacts have different epistemic roles in the way that they are used for to validate evidence. Images are not without their algorithmic and mathematical aspect, but they are defined by the role in producing them of light or sound emitted from the object imaged. Although visualisations do start off from images, there are a number of intervening steps leading to the construction of a computational mesh and finite element mesh which then serves as the basic geometry for the visualised simulation, in the form of an animated movie -- at a very distant remove from any images. The movie which is the visualisation of the simulation, is itself a rendering of the model in its predictive capacity of processes over time. The temporal aspect is crucial. Given that the mathematics is in the first place inaccessible and difficult to comprehend by non-mathematical researchers, the visualisations may be the only direct access they have to the ideas and hypotheses being proposed for later testing. Thus they play a crucial role in bridging the gap between those who speak the mathematical language, and those who do not. The modellers in the collaboration have an alternative point of access to the simulation (the mathematical model itself), even though, as Winsberg (1999) points out, even they rely on the visualisation for grasping specific features, for analysis and comparison. For the modellers, the context of the visualisations is the model and modelling process; for experimentalists, the context of the visualisations are their experiments, including instruments, techniques and so on. Thus the visualisations have different ranges of meaning for modellers and experimentalists. Despite this, the same visualisations are used by both groups in the collaboration, building up a core of shared meanings over the course of ongoing joint research projects.

This has not yet occurred for computational biology which studies multicellular processes, for reasons that become apparent when considering the second point mentioned above. The visualisations bring a qualitative observational aspect to an otherwise deeply quantitative practice, from the data requirements of mathematical modelling, to the numerical processes that occur at every stage of the development of visualisation software. Even though the history of science has often seen progress in the form of increasing mathematisation ${ }^{10}$, scientific documents have always been accompanied by illustrations and diagrams of all kinds (Lynch 1990). However, the visualisations of simulations are a very specific use of the visual in the scientific context, and this becomes very clear in the contrast between whole organ modelling and multicellular system modelling. We have seen that in the case of whole organ modelling the visualisations are shared by experimentalists and modellers, with overlapping as well as divergent meanings. Below are some typical images from a visualisation, something like 'stills' from a movie.

\footnotetext{
${ }^{10}$ See Husserl (1970) for a classic example.
} 


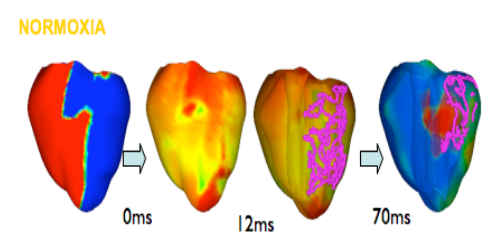

Figure 2: A set of stills from a simulation of electrical activity in the heart.

Although stylistically originally derived from textbook illustrations in order to bring to the experimentalists something they could relate to, the visualisations have not been adopted across the interdisciplinary heart modelling community. The images for the simulation of processes relating to tumour growth were also derived from textbook illustrations. An example is this image taken from a visualisation of colorectal cancer.

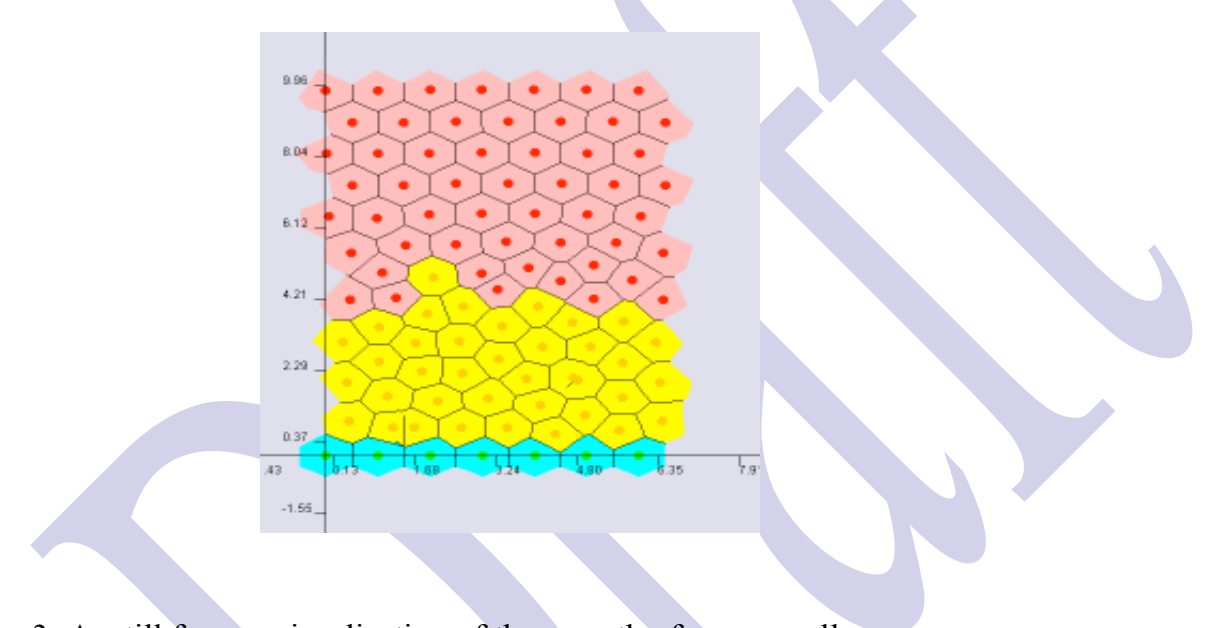

Figure 3: A still from a visualisation of the growth of cancer cells

The style is taken from the diagrams used in cell biology text books, often seen side by side with microscopial images. This particular one has adopted the style of a diagram of epithelium, which is not immediately relevant to cancer cells except for being handy for illustrating multi-cell growth. The visualisations in this domain remain rather external to the practice of both of the main disciplines involved: they are not as crucial to the work of the mathematicians studying tumour growth as they are to the computationalists studying whole organ processes, since, for example, they cannot be used for data analysis or as a tool for discovery and exploration. Their communicative and rhetorical roles is emphasised over their epistemic role. Thus the visualisations are felt to be 'for the biologists', since they help to communicate the program of research to the biologists who would otherwise not engage with the mathematics. Since the visualisations are qualitative, this is felt to reach out to the qualitative observational practices of the biologists. However, visualisations of this sort are unlikely to find many appreciators in the biological community, for reasons discussed in the next section.

Section 4: Observations and images in the context of cell biology 
The visual practice of both the computational biologists and the cellular biologists play a central role in defining their respective epistemic practice, since they are used for key exploratory, evidential and justificatory activities by both groups.

In the previous section, we have already seen that there are several points on which modellers and experimentalists diverge, and that it is necessary to bring about a convergence of interests in order to have successful collaboration. Convergence can occur around the ultimate purposes of the research (such as research for drug discovery or a similar aim), or around specific research questions and the means for answering them. For this convergence to occur, a shared mode of perception and observation emerges around artefacts such as visualisations. Convergence does not always occur and the ways in which it does not reveals further features of the general epistemic framework within which scientists operate which influence whether a shared mode of perception will emerge. In this section focus will be on those domains of computational biology which are still at incipient stages, requiring co-operation mostly between mathematicians and biologists so that the computational research will be able to take root.

Microscopy is the key epistemic instrument for biologists' exploration and discovery of their specific research fields. The microscope puts the biologist in what is experienced as direct contact with tokens of that field in the form of the samples being explored, even though the experience is in fact mediated by the microscope and the theories concerning the laws of physics which are built into it. With respect to direct access to the epistemic features relevant to the practices of each, the observation of samples through the microscope is the equivalent, in biologists' practice, of the observations of visualisations of simulations for the computational biologists. In both cases, scientists are able to access - through observation - the processes of interest to them, they are able to see the outcomes of experiments and do analysis and interpretation. This, however, is where the similarity ends since what is observed is incommensurably different.

There are several important differences between the epistemic practices of biologists and mathematical biologists. In her book Making Sense of Life, Evelyn Fox Keller expounds in some detail the differences between mathematically oriented and biologically oriented researchers. She draws attention to the highly visual nature of biological research which carries with it an epistemic outlook as well. For example, while mathematicians tend to seek theories, biologists are suspicious of theory, and give much greater priority to qualitative observation:

Traditionally, theoretical models in biology have been associated with the singling out of one particular dynamic, of one particular kind of mechanism, and the message drawn from increasing visual access underscores, once again, how far the range of biological innovation exceeds the range of human imagination. Indeed, for many, merely being able to identify the mechanisms involved suffices as an explanation. (2002: 232).

In particular, biologists do not always accept the explanatory force of theories couched in mathematical terms. Mathematicians working in the biological or physiological domain often talk of their desire to pinpoint the specific mechanisms that are causally responsible for some process. Biologists are also interested in causal mechanisms; however, what counts as a cause (or a candidate causal mechanism) is 
radically different with respect to the methodology for arriving at causal claims, and with respect to what might count as evidence in support of those causal claims. For biologists, it is often a case of 'seeing is believing'; that is, what is seen through a microscope is the ultimate arbiter as what might be the possible causes of a phenomenon. Many biologists find theoretical accounts - which by their nature go beyond what can be observed - unsatisfying; and when these accounts are couched in mathematical terms, even more so. This is not simply because they are averse to mathematical methods (although they may well be), but because the phenomena in question (as has already been discussed in section 2) are at a level of complexity which is exceedingly challenging at all levels, not least at the mathematical level. Even now, it is not infrequent to find biologists expressing opinions such as those expressed by Eric Ponder in1934:

It is futile to conjure up in the imagination a system of differential equations for the purpose of accounting for facts which are not only very complex, but largely unknown (Eric Ponder, quoted by Keller 2002: 100)

In a similar vein, the biologist Lynn Margulis ironically refers to mathematical models as "numerology"11. Thus, since biologists are more likely to be persuaded by accounts of processes which are derived from observational practices, and more likely to be suspicious of what seem to be mere speculations of processes that cannot be observed 'in vivo' but only 'in silico', the visualisations of simulations are not likely to be as compelling for biologists as they are for the computationalists. For biologists, the visualisations may seem to be theoretical constructs, which have no more plausibility than the models behind them, which, for highly complex processes, is not a high degree of plausibility. From the point of view of biologists, computational biologists underestimate the complexity of biological processes - and indeed, of what would be required to conduct the experiments required to build a plausible model. For example, one biologist interviewed commented on a failed collaboration with a computational biologist:

[The computational biologist said ] we need to know the dissociation constant of this protein, reaction diffusion equations, concentrations of proteins in the cell. That sort of stuff I don't have a clue, it would take years to get. More generally, about systems biology as a whole - how do you model networks, pathways, particular processes. Seems to me that you need to have these parameters. The concentration of your protein, how fast does it move in the cell, is it in the right location to reach with something downstream of it. And there are really only a very few biological pathways for which data like that are available.

One of the important reasons why this type of data are unavailable is the difficulty of abstraction in biological processes. Mathematicians sometimes refer to the background complexity making abstraction difficult as 'noise', whereas for some biologists, this is precisely what makes them biological. This brings into play the question of modularity and which categories or 'modules' are still distinctively

\footnotetext{
${ }^{11}$ Forum
} 
biological in character. For example, the self-styled historical biologist, Lynn

Margulis, warns against the 'Whiteheadian fallacy of misplaced concreteness' 12 and Brian Goodwin, an acclaimed mathematical, in his more recent work turned towards a more holistic approach to biology, questioning the abstraction that necessarily goes hand in hand with mathematical models as a general approach for understanding biological processes. In his view, other non-mathematical terms play a significant role in describing biological phenomena:

Brian: James [...] [y]ou used the terms 'beauty', 'harmony', 'grace'. Why not add 'wholeness' and 'health' and 'coherence'? Because you can see when microtubule organisation is coherent and when it's disorganised, and when it's disorganised it's a clue to something; some of the components are 'funny'. Now, that can be quite subtle and I would say that human intuition is bloody good at this kind of detection. ${ }^{13}$

These disagreements are also expressed with respect to the visualisations. Thus for a biologist committed to microscopy of a mode of observation, seeing a visualisation of a simulation lacks epistemic value, as well as the allure of observed processes (of which more below). Thus when mathematical biologists share with biologists visualisations such as the one in figure 3 depicting the growth of cancer cells as mathematically modelled, in a bid to share with them the qualitative practice with which they are familiar, they are unlikely to find a responsive audience. The visualisation simply looks uninteresting in the eyes of biologists who are used to seeing these diagrams alongside microscopial images or in contexts which relate to such images. The micoscopial images in their turn, are only fully significant when seen relative to the complex and wholly embodied observations of processes often by means of powerful microscopes, and even more importantly, by means of a whole panoply of techniques and tools which allow them to interact with the sample (Keller 1996).

However, mathematicians too can exhibit a great deal of scepticism regarding the 'visualisations' of biologists. For example, biologists sometimes produce animations of biological processes, such as those produced by Drew Berry ${ }^{14}$, or by those to be found on the website of Hans Clevers' Group at the Hubrecht Institute ${ }^{15}$. Typically, these visualisations are produced by means of a combination of observation, microscopy images, hand drawing, and in depth research of current knowledge of the processes depicted. These movies are used mostly for communication and education, and not for actual research. Importantly, their audience can include mathematical biologists (either in training or experienced researchers) in order to give them an idea of what the biological processes they are interested in may 'look like'. One view expressed was that it is very useful to be able to see the movies since mathematicians working in biology do not have easy access to this kind of process; the movies can help them to understand their models better by putting them in context. Other views (sometimes by the same people) were that the movies were also potentially

\footnotetext{
${ }^{12}$ Forum

${ }^{13}$ Forum

${ }^{14}$ Walter and Eliza Hall Institute of Medical Research TV: http://www.wehi.edu.au/education/wehi-tv/ (accessed $25^{\text {th }}$ October, 2009).

${ }^{15}$ Hans Clevers Group, Hubrecht Institute, Movie Animation:

http://www.hubrecht.eu/research/clevers/research.html (accessed 25th October, 2009).
} 
misleading, in that they make it appear that all the problems have been solved, that there is a complete understanding of the process. Sometimes these animations are dismissed as 'cartoons', although even this view often goes hand in hand with a distrust of what is compelling for the eye. A view that is often expressed is that the movies may be misleading in that they are seductive, and may persuade you to 'buy into' a particular way of seeing - and therefore understanding - the phenomena. One interviewee likened this to seeing the movie adaptation of a book: the picture you had in your mind may not be the same as in the movie and that may be jarring; but equally, it may override the picture you have in your mind because it is so compelling.

The question arises as to how biologists themselves view these movies and animations of biological processes. As has been stated, animations are not generally used for exploratory research themselves ${ }^{16}$; however, they can play another more emotional role for biologists, since they bring to life for them, or allow them to recognise, something which in principle, they may be able to observe. Watching the movies can resonate with the experience of observing the processes in question, and in this way, reinforce the attachment that biologists can experience to their observational practice. If there is any instrument that biologists identify with, it is the microscope - in the sense that they could not be biologists without a microscope. For example, during the course of the Forum on Scientific Method in Biology, James Wakefield addressed the question of scientific method in biology first and foremost by describing the ritual of setting up a session with the microscope:

The affinity of a cell biologist to his microscope shouldn't be underestimated. Although, I understand the physics of how a microscope works, it's not the physics that interests me. It's using the microscope as an extension of my eyes in order to perceive the innermost workings of a cell. In order to do that, I guess it becomes quite ritualistic. So, I take samples, cells, tissues, and I prepare them in a certain way. And then I'll go to my little microscope room, little dark room, pull the curtain, sit down on my seat, and then I 'll go through a series of well-trod steps; I'll take the cover off the microscope, I'll put this little light on and that little light on, the $\mathrm{x}$, y stage. And then when I 'm comfortable, when I'm feeling 'in the zone', I'll put the sample on there and I'll take a very cursory glance around what's on that microscope slide. And instinctively I know whether it's a good sample or a bad sample, whether the fixations worked properly, whether the stains worked properly, whether there are any differences between control and treated sample $[\ldots]^{17}$

Similar rituals are not uncommon with other cell biologists. A closer examination reveals that it is not simply the instrument as such; rather, it is the observational situation comprising person-microscope-sample and the entire set of interactions around it that is so strongly emblematic of what it is to be a biologist. And the reason for its being emblematic is the direct access to biological processes that the situation is experienced as affording.

\footnotetext{
${ }^{16}$ The hand-drawn depictions of cells by biologist David Goodsell, blur this boundary.

${ }^{17}$ Forum
} 
Cell biologists experience a strong identification with the instruments, tools and techniques of microscopy. Throughout its history, the microscope has caught the imagination of natural history enthusiasts, promising a means for 'exploring the inner labyrinths of nature' (Harley Warner 1982) but it took some time for it to become an instrument for serious scientific pursuit. The turning point occurs when it became possible not only to look through a microscope passively, but to interact with it and with the sample. As Evelyn Fox Keller shows, the professional biological gaze through the microscope came into being when the object is no longer merely looked at but also manipulated. This is especially important in establishing that what is seen through the microscope is real and not an artefact of the microscope. Keller writes:

[...] once the microscope was joined with the manual manipulations of an experimental biology - marking, cutting and dissecting under the scope - and the interdependency of hand and eye previously reserved for the naked eye was extended into the microscopic realm, the microscope became a reliable tool for veridical knowledge (1996: 112)

What is considered to be real in both everyday and scientific contexts is very closely connected to perceptions of causality. The interactions that are possible with observed samples - isolating one part from others, marking with dye, separating, extracting, injecting, etc - and in all instances watching and following with the eye what happens following each of these interventions - creates a context in which causality can be experienced, in the first instance, in the very interactions themselves, including the mistakes. Ian Hacking's description of learning to observe by active doing, is vivid:

The conviction that a particular part of a cell is there as imaged is, to say the least, reinforced when, using straightforward physical means, you microinject a fluid into just that part of the cell. We see the tiny glass needle - a tool that we have ourselves hand crafted under the microscope - jerk through the cell wall. We see the lipid oozing out of the end of the needle as we gently turn the micrometer screw on a large, thoroughly macroscopic plunger. Blast! Inept as I am, I have just burst the cell wall, and must try again on another specimen (1983: 189-190)

As Keller points out, this 'making real by touch' is the entry way to the scientific realm of causal efficacy, or 'making things real by using them to effect change in other things we know are real' (1996: 114). It is clear that given this close interaction between seeing anything that is of scientific interest, and interacting through manipulations of different types through techniques made possible through yet other tools and technologies (micropipettes, microneedles, etc), that which is seen is an artefact, in the sense that it is constructed in the configuration of viewers, instruments, and theories that every viewing episode sets in place. The question arises as to why this still counts as direct access to the biological process?

Compare it for a moment to the visualisation that renders the outcome of a simulation. This too is a highly constructed viewing episode made possible by a particular configuration of viewers, instruments and theories. The computational biologist is as interactive as the biologist with their visualisations: for example, they can halt them, 
adjust parameters and see what happens, alternate views, etc. The visualisations used for computational biology also include social interactions, which is crucial for this highly cooperative and collaborative research area. The visualisations as material artefacts are fully incorporated (in the fullest sense of the word) into research events such as seminars and workshops, where they are displayed, animated by gestures, and used as media for social interaction. Physical interactions can also occur through the computational instrumentation that render the visualisations, or that analyse and process data: the software as well as (or through) the screen, the mouse and keyboard. Computational biologists experience their visualisations as compelling too; any why not, since through them they gain [or seem to gain] direct perceptual access to that which fascinates them: mathematical constructs. During the course of the interaction with the visualisations, the abstract nature this construct recedes into the background. As Araya puts it:

[...] implicit in our encounter with the v-molecule on the screen is the model of a molecule. In a sense, the v[isualisation]-molecule is 'parasitic' on the model behind it: From the model it borrows 'meaning', which makes possible our encounter with it' without the model the v-molecule would decay into a meaningless collection of surfs. But once they come alive for us, that to which they refer tends to disappear while they become the focus of attention. We find these beings much more congenial to us than their referents, because we can create them, see, manipulate, and 'think' with them (Araya 2003:75)

In the observational situations of biology and computational biology there are therefore several structural features in common: a high degree of what we can call 'fusion' between observers and instruments directed at processes to be made sense of. The instruments combine those which render a visual artefact to the gaze, and those which enable interaction and manipulation. None of these elements can be isolated from the others in the observation of the process in question: there is nothing to be seen without the intervention of viewers and instruments. The englobing context is that of the broader epistemic and institutional context in which the entire configuration of viewers, instruments and processes occurs, and from which it gains meaning. There is no innocent eye, there is no given object, there is no neutral instrument.

Finally, the two practices are distinguished by different forms of social configurations. There is a core of inalienable 'alone-ness' at the heart of cell biologists observational practice ${ }^{18}$, and of this there is no equivalent in the visual practice of the computational biologist. Visualisations, as opposed to microscopymediated observations, are all public artefacts existing in the intersubjective world in the form of images, stills, movies, animations, and so on. Biologists do share their images and movies taken during microscopy sessions, and they do use these for exploratory research as well as to provide evidence for their claims. However, this does not replace actual observations, since they tend to flatten the process as observed in $3 \mathrm{D}$ into a $2 \mathrm{D}$ representation. A typical example is shown in figure 4 . Therefore they are experienced as being secondary representations of the 'real thing', and in the evidential chain, it is in further observations carried out that other scientists will be

\footnotetext{
${ }^{18}$ At least so long as only one person at a time can look down a particular microscope at a particular sample.
} 
able to test that evidence. These are observations which they themselves will carry out, which contrast on one hand with looking at images or movies made by others the processes of which they could, in principle, carry out themselves; and on the other with considering the inputs of collaborators from other disciplines which they cannot carry out themselves, but for which they rely on interdisciplinary teamwork. The work of full-fledged computational biologists such as heart modellers instead, is very much a matter of interdisciplinary teamwork, where team members can undertake specific sections or aspects of the research, but no-one of them can master all of the techniques involved. This does not make microscopial observation an activity which is not socially mediated, since without being immersed in a community of biologists, observation would not yield much; however it does mean that distinct episodes of observation can put biologists in a position of feeling that they are observing something for themselves, without having their vision directed by others. This is a further point of resistance to sharing visual practices around visualisations of modelling and simulations, since the publicity of these artefacts does not give access to a private moment of 'seeing for oneself' an event that is actually occurring down a microscope.
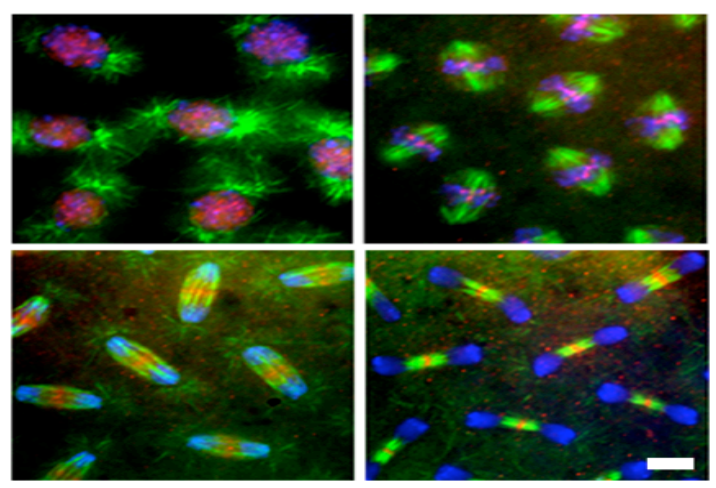

Figure 4 A typical microscopial image using fluorescent microscopy

\section{Conclusion}

In some instances, computational biology is akin to the shared practices passed on from professor to student in the archaeological dig. Inter-disciplinary researchers learn from each other and come to share some aspects of each others' visual practices. In other instances, it is akin to the lawyers' use of video evidence in the Rodney King case: using a display of prowess with images in order to try to persuade potential collaborators on board. But just as in that case, their success is not guaranteed, and the vision that they offer of events or phenomena may be resisted by their audience. The audience shares neither the vision, nor the techniques and practices for shaping it as desired by the It is these cases of contested vision that show the limits of social sharing of practices for the shaping of events and phenomena across communities.

Gaps, fissures and breakdowns in sharing visual practices show the limits of what can be accomplished by the social sharing of these practices alone, and show that sharing, where it does occur, is predicated upon a much wider set of factors. Faltering or even failing inter-disciplinary collaborations where there are competing views on the same field are good places to examine these limits. The question when can practices be shared further relates to other questions such as: who does the observing, with what 
activities, techniques, instruments, in what contexts and for what purposes? All of these converge on the question: 'what is seen or observed?'. The observations of in silico experiment and in vivo experiment are undoubtedly both constructions, but this still leaves plenty of scope for real differences regarding the epistemology and ontology of the broad field of biology. Whether progress is made in the more intractable sub-domains of computational biology depends on whether the translation can be made between models and simulations on one hand and the type of experimental data obtained through the specific techniques characteristic of that subdomain on the other. Visual practices - including practices of observation - are a central aspect of these techniques, since they are often one of the primary means through which evidence is constituted within the sub-domain. Evidence interweaves interactions between people, instruments and the entities observed, be they simulated or 'real', creating a web that is at the same time social, instrumentational and ontological. Sociological theories such as Actor Network Theory do attempt to include the objects of study as potential shapers of scientific knowledge, or as playing their role in a network of inter-relations between different factors. When broadened out in this way, it begins to become more apparent why visual practices cannot be shared across some domains without a concomitant change or even sacrifice of the very thing that is felt to define that domain of enquiry: the thing that is studied, about which researchers want to know and understand more, towards which their enquiry is directed. There is a personal and subjective level to this in that what is studied and how it is studied defines not only the domain of enquiry but oneself as scientist in relation to it. It is this, perhaps that accounts for the resistance to sharing visual practices: not simply because they are different ways of doing, but because they change the domain of enquiry, and in so doing, also potentially change the researcher, or make a demand for change at a very deep level of the way that researchers experience their own identity in a domain.

Between the biological sciences considered in this paper, a central difference in the nature of the visualisations, images and microscopial observations is the ontology of the that towards which the scientists' visual practices are directed. No doubt neither of these are unadulterated natural objects, and they are both constructs of practices, instruments and theories. In fact, they are even both models, since the samples observed through microscopy are generally of animal models, such as drosophila, sea urchins or worms. But the way in which they are constructed, the history that give rise to them, and, importantly, the role of the biological object in them, is substantially different. In the process of modelling and simulation, the biological object is external, at least to the observation of the visualisation that renders the process. What is observed is the model; it functions, as I have said, like a concretisation of an otherwise abstract entity. It is not dissimilar to Kant's proposal that metaphors and other images 'body forth' or make concrete an abstract idea, and are, for that very reason, an object of aesthetic pleasure - and hence, to continue in an un-Kantian way - of attachment and identification ${ }^{19}$. The biological object or process which is modelled acts as an external constraint; it is an external validator or data provider, not that which is seen or embodied in the visualisation. What is observed through the microscope is instead the biological object. It is useful to turn to the section in

\footnotetext{
${ }^{19}$. In the production of aesthetic ideas, genius 'attempts with the aid of an imagination which emulates the display of reason in its attainment of a maximum, to body forth to sense [rational ideas] with a completeness of which nature affords no parallel' (Kant 1952: 176-7). Aesthetic ideas serve 'as a substitute for logical presentation' for rational ideas (1952:177).
} 
Merleau-Ponty's Phenomenology of Perception (1962) which deals with 'The Thing and the Natural World'. Merleau-Ponty's account of the relation between perceivers and 'the thing in the natural world' offers a framework for thinking of the relation between subjects and objects as one of inter-constitution. Merleau-Ponty writes of the relation between perceiver and perceived as one of communication and dialogue, where each completes the other. In this relationship, the objects of science are far from being 'docile objects' (Lynch 1985: 43). Even though natural objects become objects for us through a certain organisation and arrangement of their sensible aspects (they have, as Merleau-Ponty puts it in Visible and Invisible (1968) an opening onto subjects, as subjects have to objects, in a reversible relation of intertwinement), at the same time we cannot ever know them entirely, they can always surprise us. Even more, we might say that scientific vision offers an experience like Klee's, of objects that 'look back' and with which the eye and hand are interwoven: 'The eye [...] is that which has been moved by some impact of the world, which it then restores to the visible through the traces of a hand' (1993: 127). In this relationship with natural objects, the moves we make in the dialogue are completed by the object and vice versa.

... every perception is a communication or communion, the taking up or completion by us of some extraneous intention or, on the other hand, the complete expression outside ourselves of our perceptual powers and a coition, so to speak, of body with things. (1962: 320)

Perceiving things is in a sense to live them, to have them lodged within oneself in one's orientation towards the world:

In order to perceive things, we need to live them ... To 'live' a thing is not to coincide with it ... Our problem, therefore, becomes clear. The perceiving subject must, without relinquishing his place and his point of view, and in the opacity of sensation, reach out towards things to which he has, in advance, no key, and for which he nevertheless carries within himself the project, and open himself to an absolute Other which he is making ready in the depths of his being. (1962: 325-6) ${ }^{20}$

Within the bounds of this paper, it is not possible to delve further into the ontology of computational simulations and visualisations on one hand and of microscopial observations on the other. A fuller account is necessary. My aim has been to show that the sociality of visual practices - the fact of their being shared by communities is not sufficient to account for what is seen through those practices; in particular, it

\footnotetext{
${ }^{20}$ The details of the implications of this account - intended for basic experience and not for science as such - cannot be broached here (See Rouse (2004)). Of course it makes a difference that for MerleauPonty, science is a 'second-order expression' relative to a 'basic experience of the world', although 'second-order' here does not mean that it is any less intensely lived. On the contrary, if anything, we might suppose that scientists live the relation to their objects and artefacts more intensely than basic experience, and the experience of being in a dialogue with these, of interrogating them with ones eyes and other senses, and through one's instruments more keenly felt, and at any rate, no reason to think that the ontology of these domains is a diminished version of the ontology of basic experience. There is also a particular significance to the biological sciences, as evident in Merleau-Ponty's elaboration of his ontology of the flesh in his thoughts on the life sciences, in Nature (1995).
} 
does not account for the resistance to come to see as others do. The alternative framework that has been offered is one whereby scientific viewers and scientific objects inter-constitute each other and define a world with a specific ontology of subject and of object. This implication of the scientist in her world makes it little wonder then that there is such an attachment to particular ways of seeing in specific sciences, and so much resistance at coming to see as others do beyond a certain limit. The limits of sharing are defined by the limits of the worlds that they occupy, and crossing these limits is not so much sharing, as transforming those worlds.

\section{References}

Araya, A. 2003. “The Hidden Side of Visualisation.” Techné 7/2: 27-93.

Baigrie, Brian S. 1996. Picturing Knowledge. Historical and Philosophical Problems Concerning the Use of Art in Science. Toronto: University of Toronto Press.

Burton, R. et al. 2006. "Three-Dimensional Models of Individual Cardiac Histoanatomy: Tools and Challenges.” Annals of the New York Academy of Sciences 1080: 301-319.

Byrne, H.M., Alarcon, T., Owen, M.R., Webb, S.D., and Maini, P.K. 2006. "Modelling aspects of cancer dynamics: a review."Philosophical Transactions of the Royal Society 364: 1563-1578.

Carusi, A. 2008. "Scientific visualisations and aesthetic grounds for trust." Ethics and Information Technology. 10: 243-254.

Carusi, A., Rodriguez, B., Wakefield, J. et al (2009) Forum for Scientific Method in Biology [details to be supplied once the transcription has been archived]

Galison, P. 1997. Image and Logic: A Material Culture of Microphysics. Chicago \& London: University of Chicago Press.

Gavaghan, D., Garny, A., Maini, P., and Kohl, P. 2006. "Mathematical models in physiology." Philosophical Transactions of the Royal Society 364: 1099-1106.

Goodwin, C. 1994. "Professional vision.” American Anthropologist 96/ 3: 606-633.

Goodwin, C. 1997. "The Blackness of Black: Color Categories as Situated Practice.” pp. 111-140 in Discourse, Tools and Reasoning: Essays on Situated Cognition. Edited by Lauren B. Resnick, Roger Säljö, Clotilde Pontecorvo, \& Barbara Burge. Berlin, Heidelberg, New York: Springer

Hacking, I. 1983. Representing and Intervening. Cambrige: Cambridge University Press.

Harley Warner, J. 1982. “'Exploring the Inner Labyrinths of Creation' Popular Microscopy in Nineteenth-Century America," Journal of the History of Medicine and Allied Sciences 37:1

Hunter, P.J. \& Borg, T.K. 2003. "Integration from proteins to organs: the Physiome Project." Nature Reviews 4, March 2003: 237-243. 
Husserl, E. (1970) The Crisis of the European Sciences and Transcendental

Phenomenology. Including The Origin of Geometry. Trans. by David Carr. Evanston: Northwestern

University Press.

Jones, C.A. and Galison, P. 1998. Picturing Science, Producing Art. New York, London: Routledge.

Kant, I. 1952 Critique of judgement. Trans by J.C. Meredith. Oxford: Clarendon.

Keller, E. F. 1996 “The biological gaze.” Pp 107-121 in FurureNatural. Edited by George Robertson et al. London: Routledge.

Keller, E. F. 2002. Making sense of life: Explaining Biological Development with Models, Metaphors and Machines. Cambridge, Mass. \& London, England: Harvard University Press.

Kohl, P., Noble, D., Winslow, R.L., Hunter, P.J. 2000 "Computational modelling of biological systems: tools and visions." Philosophical Transactions of the Royal Society, 358, 579-610.

Komarova, N. 2005. "Mathematical modelling of tumorigenesis: mission possible." Current Opinion in Oncology. 17/1: 39-43.

Latour, B. 1986. "Visualization and Cognition: Thinking with Eyes and Hands". Knowledge and Society. 6: 1-40.

Lynch, M. 1985. "Discipline and the material form of images: An analysis of scientific visibility". Social studies of science. 15: 37-66.

Lynch, M. 1990. "The externalized retina: Selection and mathematization in the visual documentation of objects in the life sciences." Pp 153-186 in Representation in Scientific Practice. Edited by Lynch, M. \& Woolgar, S. Cambridge MA; London: MIT Press.

Mascord, M., Jirotka, M., Carusi, A. 2007. Integrative Biology VRE. Digital Pen Evaluation Report. http://www.vre.ox.ac.uk/ibvre/IBVREAnotoEvaluation.pdf

Merleau-Ponty, M. 1962. Phenomenology of perception. Translated by C. Smith. London: Routledge \& Kegan Paul.

Merleau-Ponty, M. 1968. Visible and Invisible. Translated by A. Lingis. Evanston, Northwestern University Press.

Merleau-Ponty, M. and Séglard, D. 1995. Nature. Course Notes from the College de France. Translated by R. Vallier. Evanston, Northwestern University Press.

Merleau-Ponty, M. (1993) "Eye and Mind." Pp 121-149 in The Merleau-Ponty Aesthetics Reader: Philosophy and painting. Edited by G.A. Johnson. Evanston: Northwestern University Press.

Noble D. 1962. "A modification of the Hodgkin-Huxley equations applicable to Purkinje fibre action and pacemaker potentials." Journal Physiology 160: 317-352.

Plotkowiak, M., Rodriguez, B., Plank, G., Schneider, J., Gavaghan, D., Kohl, P., Grau, V. 2008. "High Performance Computer Simulations of Cardiac Electrical Function Based on High Resolution MRI datasets." Computational Science - ICCS 2008. 5101: 571-580. 
Rouse, J. 2005. "Merleau-Ponty's Existentialist Conception of Science.” Pp 265-291 in Cambridge Companion to Merleau-Ponty. Edited by T. Carman and M.B.N. Hansen. Cambridge: Cambridge University Press.

Sharrock, W. and Coulter, J. 1998. “On What We Can See.” Theory and Psychology. 8/2: 147-164.

Van Leeuwen, I.M.M., Edwards, C.M., Ilyas, M., Byrne, H.B. et al. 2007. "Towards a multiscale model of colorectal cancer.” World Journal of Gastroenterology. 13/9: 1399-1407.

Welsh, E., Jirotka, M., Gavaghan, D. 2006. "Post-genomic science: Cross-disciplinary and large-scale collaborative research and its organizational and technological challenges for the scientific research process." Philosophical Transactions of the Royal Society. 364: 1533-1549.

Winsberg, E. 1999. "Sanctioning Models: The Epistemology of Simulation." Science in Context. 12/ 2: 275-292. 